\title{
General Law of the Universe and Unity of All Universal
}

\section{Forces}

\author{
Jinzhong Yan \\ Changsha Jiageer Machinary Manufacture Co., Ltd., Pingtang Town, Changsha City 410208, Hunan Province, China
}

\begin{abstract}
All life forms in the universe have four-seasonal structure, four-seasonal movement, four gradations, and rise and fall of four gradations. This is the common feature of all life forms in the universe and the general law of the universe. The unity of the universe includes the unity of all universal forces which consist of two kinds, physical force and life force. The former includes bonding force, strong interaction, quark confinement, weak interaction, electromagnetic interaction and gravitational interaction and is also called four-seasonal structure. The latter lies inside the life forms, combines life beings and pushes the four-seasonal movement and rise-fall movement inside life forms. Life force includes transverse four-seasonal force, longitudinal four-seasonal force, four-gradation force, life being structural force and four-gradation rise-fall force. All these forces are united to form a complex life force system. Life force is also a compound, integral, long-range and automatically-closed four-seasonal force. Life being structural force is a force that connects all parts of a life being to make it an integral being. In the universe, life being structural force is an invisible three-dimensional net and a rigid structure and movement. It endows all heavenly bodies with a highly orderly structure and movement. Morality is the dominator of the universe. The whole universe is a moral system and all things in it have morality. Morality is also presented as a force, namely moral force, including rise force and fall force. The unity of all forces in the universe is the general law of the universe.
\end{abstract}

Key words: Universe, general law, various forces, unity.

\section{Introduction}

Considering the universe as a life form is the precondition to propose GUT (Grand Unified Theory). All life forms in the universe have four-seasonal structure, four-seasonal movement, four gradations and rise and fall of four gradations [1]. That is to say, all life forms have the same structure and movement. Each life form is in a different gradation due to different internal force. All life forms in the same gradation form a four-seasonal structure to do four-seasonal movement. Life forms of different gradations can rise by increasing internal force or fall by decreasing internal force, forming a dynamic balance between rise and fall. This is just the primary feature of the universe and the general or overall law of the universe.

GUT includes many aspects of the unity of the

Corresponding author: Jinzhong Yan, senior engineer, research field: disciplinary sciences. universe, naturally including the general law of the universe and the unity of all forces of the universe. The unity of all universal forces is only one part of universe unity but not the entire content.

First, we should make clear how many forces are there in the universe. Modern physics only researches physical force not including life form force the major force of the universe. Physicists call the unity of strong interaction, weak interaction, electromagnetic interaction and gravitational interaction GUT [2]. This is not appropriate. However, life is made up of materials, so physical force is the base of life form force.

The universe is a life form. Life form force makes up the majority of universe force. The four-seasonal structure, four-seasonal movement, four-gradation structure and rise and fall of four gradations of universal life forms are all dominated and controlled by the life force. Finding all the life form forces of the universe according to the general universal law, we 
are able to realize the unity of all universal life forces.

\section{Identity Principle of Universal Life Structure and Movement}

According to Ref. [1], all life forms in the universe have four-seasonal structure, four-seasonal movement, four gradations and rise and fall of four gradations. That is to say, all life forms in the universe have the same structure and movement. The only difference among them lies in moral internal force [3]. This is the identity principle of universal life structure and movement.

Different life forms with different moral internal forces are in different state space-times. For example, humans and animals are in different state spaces and plants and bacteria belong to different state times. Human states are divided into three-dimensional humans, two-dimensional humans, one-dimensional humans and zero-dimensional humans [4]. Morality is not just force but also moral seed which brings cause and effect. Life forms with different moralities enter different life spaces and bring causes and effects accordingly. This is what is called samsara in religion [3].

\section{General Law of the Universe}

\subsection{General Law of the Universe}

According to the identity principle of life structure and movement, we can show the structure and movement of life forms in Fig. 1. It is the structure and movement pattern of all life forms in the universe and thus the general law of the universe.

Four states coexist in life forms and there are also four states coexisting in the universe. In Fig. 1, just as Laozi argued "Tao generates one, one generates two and two generate three" [5], he also deduced "follow to become mortals; reverse to become immortals". The universe, galaxies, creatures, society and man-made world all follow this procedure $[3,6]$, just as Table 1.

\subsection{Universe Unity Includes the Unity of Various Universe Forces}

Fig. 1 is not just the general law of the universe but also the universal unity pattern [1]. This pattern includes various unities in the universe, naturally including the unity of various universal forces.

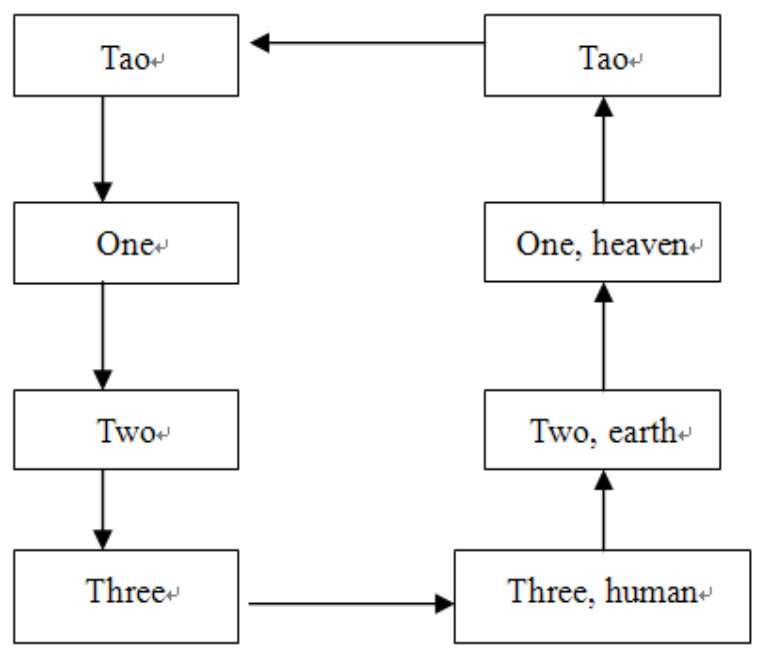

Fig. 1 General Law of the Universe.

Table 1 Cycle Evolution of Galaxies and Humans.

\begin{tabular}{|l|l|l|l|}
\hline Life state & Galaxy's evolution & Human's evolution & Change tendency \\
\hline Tao, zero & Zero-dimension, chaos & Buddhism and Taoism & Fall \\
\hline One & One-dimension, nebula & Buddha & Fall \\
\hline Two & Two-dimension, star forming & Arhat & Fall \\
\hline Three & Three-dimension, planet forming & Mortal, most corrupt & Fall \\
\hline Three & Three-dimension, spiral galaxy & Mortal, become to awake (now) & Rise \\
\hline Two & Two-dimension, elliptical galaxy & Arhat & Rise \\
\hline One & One-dimension, quasar & Buddha & Rise \\
\hline Back to Tao, zero & zero-dimension, chaos & Buddhism and Taoism & Rise to fall \\
\hline
\end{tabular}




\section{Definition, Property and Classification of Force}

\subsection{What Is Force?}

In my opinion, force is an ability to combine, move and change things and one of the state features of things [7]. It is a kind of strength in form of energy. According to the definition, we can divide force into binding force, structural force, moving force, changing force and decomposing force. The formation, movement and change of the universe are all caused by force. Force exists at all times and in anywhere. All things have force.

\subsection{Structure, Forces and Energies of Objects Are Identical}

According to concept of state space-time [7], everything in the universe has state feature, which includes structure, force, energy, etc. The structure, force and energy of all things are unified. The structure of them determines their force and energy. Namely, the structure, force and energy of all things are identical.

\section{3 Classification of Force}

Force consists of physical force and life form force. Physical force is an individual force and a force in physical aspect, while life form force is a systematical force.

\section{All Physical Forces United into Four-Seasonal Structure}

The unity of physical forces includes various physical forces: bonding force, strong interaction, quark confinement, weak interaction, electromagnetic interaction, universal gravitation and universal repulsion [1]. It is not just the unity of strong interaction, weak interaction, electromagnetic interaction and gravity, as the physicists have said.

In the four-seasonal system [8], the spring mechanism is for material absorbing and also an one-dimensional process linear force and the winter mechanism is for material releasing and also a reverse one-dimensional process liner force. The spring mechanism and the winter mechanism form an attraction-repulsion confinement which corresponds to the quark confinement. The summer mechanism is an energy mechanism and also a two-dimensional process membrane force which completely corresponds to the strong interaction of exchanging $\pi$ meson. The autumn mechanism is a material composition three-dimensional mechanism and a three-dimensional force which corresponds to bonding force. The controlling mechanism of four seasons is a zero-dimensional process. It's the DNA spiral motion and corresponds to neutrino. All attractions react to one another to combine all kinds of materials. The electromagnetic interaction exists among matters [1, 9]. These six forces are combined to form the four-seasonal structure. See Fig. 2.

From the above, we can conclude that the six physical forces are combined to form the four-seasonal structure. Each force is presented as attraction and repulsion [9], namely composition and decomposition. Thus Fig. 2 is also a double four-seasonal unity $[6,9]$. This is just the unity of various physical forces. However, this four-seasonal structure is not a life form yet because there is no invisible system.

\section{Major Features of Life and Various Life form Forces}

What we often called "vitality" is the force of life forms. It is different from physical force.

\subsection{Life Form Force and Its Variety}

It exists within life forms. It combines life forms and pushes them to do four-seasonal movement and rise-fall movement. We call it life form force because it is shown as life energy.

The basic structures of life forms are the four-seasonal structure (transverse and longitudinal) and 


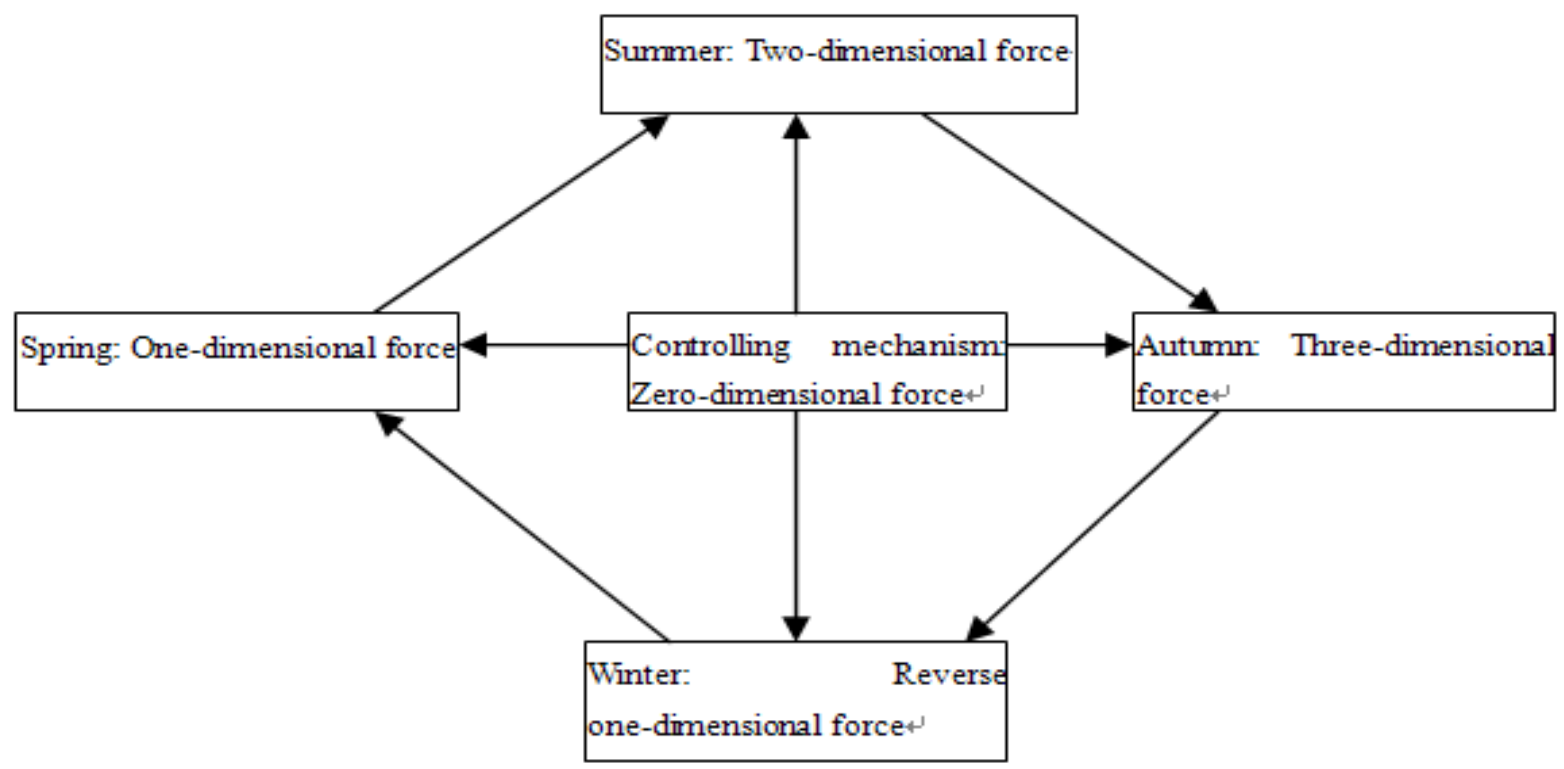

Fig. 2 Four-seasonal structure of physical forces.

four gradations. These structures and movements all possess force. Accordingly, life form forces include the four-seasonal force (transverse and longitudinal) and four gradation forces. Besides, there is the integral life form structural force as well as the morality rise-fall force within four gradations controlled by thought and morality. All these forces form a complex life form force system.

\subsection{Features of Life Form Force}

According to the features of life forms, we can find the following features of life form force: 1) It is the four-seasonal combination of all physical forces and thus a combined force. 2) It is an integral force and long-range force. Small life forms have small force ranges while large life forms have large force ranges. 3) It is a closed cycle force. Thus life form force is a four-seasonal endless cycle. 4) Life form force movement is an automatic and intellectual four-seasonal movement. 5) The forces of all life forms (creatures, the solar system, galaxies and the universe) are unified because they have the same structure and property. The forces of smaller life forms are contained in the forces of larger life forms and they react to each other.
6.3 Transverse Four-Seasonal Structural Force of Life Forms

Life forms have transverse four-seasonal structure and four-seasonal movement and they also have double four-seasonal systems and various transverse four-seasonal systems, thus having transverse four-seasonal force.

\subsection{Longitudinal Four-Seasonal Structural Force of Life Forms}

The longitudinal four-seasonal development of life forms is driven by their longitudinal four-seasonal system. Thus, life forms have longitudinal four-seasonal force and energy, which is represented as 60 Jiazi (a cycle of 60 years) in traditional Chinese science [10]. Inside life forms, there is changing mechanism [6] which along with 60 Jiazi system is the force and energy to drive longitudinal four- seasonal movement inside life forms.

\subsection{Four Gradation Force of Life Forms}

There is invisible system within the four gradations of life forms: zero-dimensional-one chaos hole, energy hole and energy cave, one dimensional-four pubic lines, two dimensional-eight energy lines, 
three dimensional-twelve energy channels [11]. They are not only forces but also structures and energies. All the structures and forces of all gradations are endless closed cycle structures.

\section{Invisible Life Structures of the Universe and Life Form Structural Forces}

Life form is made up of many subsystems. It is an integral structure, a three-dimensional network, connecting all subsystems. All life forms in the universe, including large life forms (galaxy clusters and galaxies) and small life forms (plants and bacteria), have their own integral structures. These integral structures are visible or invisible and are represented as life form structural forces.

\subsection{Visible World and Invisible World}

The visible world is the world that we can see. The invisible world cannot be seen. They are opposite and are related to the internal force of the viewers-human beings. When the internal force of a viewer is low, he can only see the materials with low forces but not those with high forces. When his integral force is high, he can see the materials with low forces as well as those with high forces.

The visible world is a low-force world, such as the visible heavenly bodies and objects. It has the features of three dimensions, low forces and slow moving speeds. The invisible world is a high-force world or a delicate world, such as the quantum world, the spiritual world, human thoughts and the energy channel system. It has the features of high force, fast moving speed and being delicate and invisible.

\subsection{Invisible Life Form Structure of the} Universe-Three-Dimensional Heavenly Network

The universe is a life form with orderly structure, movement and change. According to modern astronomy, the distances between heavenly bodies and their orbits are fixed. There are nine planets in the solar system: the Mercury, the Venus, the Earth, the
Mars, the Jupiter, the Saturn, the Uranus, the Neptune and the Pluto, whose orbits around the sun are all fixed. These nine planets are doing four-seasonal breathing movement in their own orbits and have rigid connections with the sun. This shows the solar system (the entire universe included naturally) has a highly orderly structure and movement change.

We also find that there is a cubic invisible structure outside the earth, the moon, the sun and the solar system and that there is an invisible energy axle connecting each of the nine planets with the sun [12]. Each of the nine plants has an ecliptic plane which is a three-dimensional structure. Apart from the nine planets, there are many heavenly bodies that surround the sun in the solar system. All of them have their own invisible structures and rigid axles. For example, the earth's ecliptic is invisible but the human ecliptic - the spine-is visible [11]. From this, we can conclude that the invisible structure of the universe does exist.

Various huge heavenly bodies in the universe gather and keep a distance and have rigid structures and movements. This is because there is strong force structure to regulate them. There is no doubt that this structure is invisible. All heavenly bodies in the universe are formed and organized automatically according to this structure and are restricted by it when they are moving.

The invisible universal life structure is an endless three-dimensional heavenly network and we are all living in this network.

\subsection{The Invisible Life Structure of the Universe Is Represented as Life Structural Force}

The invisible life structure of the universe is an invisible heavenly network and everything is controlled by this network [5]. This invisible life structure is also a huge life form which has the force to create any visible life forms and is represented by life structural force. There is no doubt that the life structural force is universal and has effects on all materials. This invisible life form also has breathing 
movement, thus generating attraction and repulsion, namely universal gravity and universal repulsion.

\section{Morality System and Morality Force of the Universe}

Tao and De are the basic concepts of traditional Chinese science. The Chinese culture is the culture of morality. The Chinese civilization is the civilization of morality. The basis of the Chinese morality culture is shown in The Book of Changes, Four Classics of Huangdi, The Inner Canon of Huangdi and Tao Te Ching, as well as the Chinese characters and traditional Chinese medicine. The universe is a moral system. As we often say "Morality Appeal", morality has its appeal which is also a force. How it goes?

\subsection{The Definition of Morality}

Taoists believe Tao is the essence of the universe, the general law of the universe and the origin of everything. De is the expression of Tao and its symbol. Everything in the universe originates from Tao and De [5]. Tao is zero and De is one [13].

Tao and De are everywhere. In terms of material science, neutrino is zero and quark confinement is one [7]. That is to say, the material of Tao is neutrino and the material of De is proton. Neutrino has the capacity to permeate into everything in the universe. It is "as large as to have no outside bound and as small as to have no inside part". Proton is quark confinement and has the capacity to combine everything in the universe. Therefore, zero and one or Tao and De are the most powerful forces in the universe. The two combine and control everything in the universe.

As the material of Tao-neutrino and the material of De-proton permeate at every single corner of the universe; the universe is filled with the energies and materials of Tao and De, giving everything in the university morality. Everything originates and changes according to morality and is controlled by it, too. Thus, morality is the dominator of the universe and the universe is a moral system.

\subsection{Moral System of the Universe}

Everything in the universe has morality. Different things have different morality contents. According to the contents of morality, things are divided into four gradations: Tao, one, two, and three. Life forms of different gradations have different morality energies. For example, thought offices differ due to different thought elements in human's brain. See Table 2.

The universe is a four-seasonal system controlled by Tao and De. Tao and De dominate the four seasons and are hidden in the four-seasonal system after scattered. The four-seasonal system consists of five elements: wood, fire, earth, metal and water [14]. As what has been mentioned above, everything has morality and can create morality. Thus, the five elements can create five moralities: mercy, courtesy, trust, justice and wisdom [5]. According to their amounts of energy, they are lined as mercy, justice, courtesy, wisdom and trust. Of the five morality energies, mercy has the highest energy and is the closest to Tao De while wisdom has the lowest energy and is the furthest from Tao. Trust is the carrier of mercy, justice, courtesy and wisdom. When one loses his mercy, justice, courtesy and wisdom, he loses his trust. He cannot be saved any more.

Therefore, the moral system includes Tao, De, mercy, justice, courtesy, wisdom and trust and has four gradations: zero, one, two, and three. Tao is zero.

Table 2 Thought and moral system inside life forms.

\begin{tabular}{|l|l|l|l|l|}
\hline Moral system & Invisible force and energy of life forms & Thought element [11] & Thought office & Moral system \\
\hline Zero & Chaos hole, energy hole, energy cave & True self & Nine-grid pattern [11] & Tao \\
\hline One & Four pubic lines & Positive self & Brain core layer & De \\
\hline Two & Eight energy lines & Negative self & Brain mass layer & Mercy \\
\hline Three & Twelve energy channel & Front six cognitions & Brain cortex & $\begin{array}{l}\text { Justice, courtesy and } \\
\text { wisdom }\end{array}$ \\
\hline
\end{tabular}



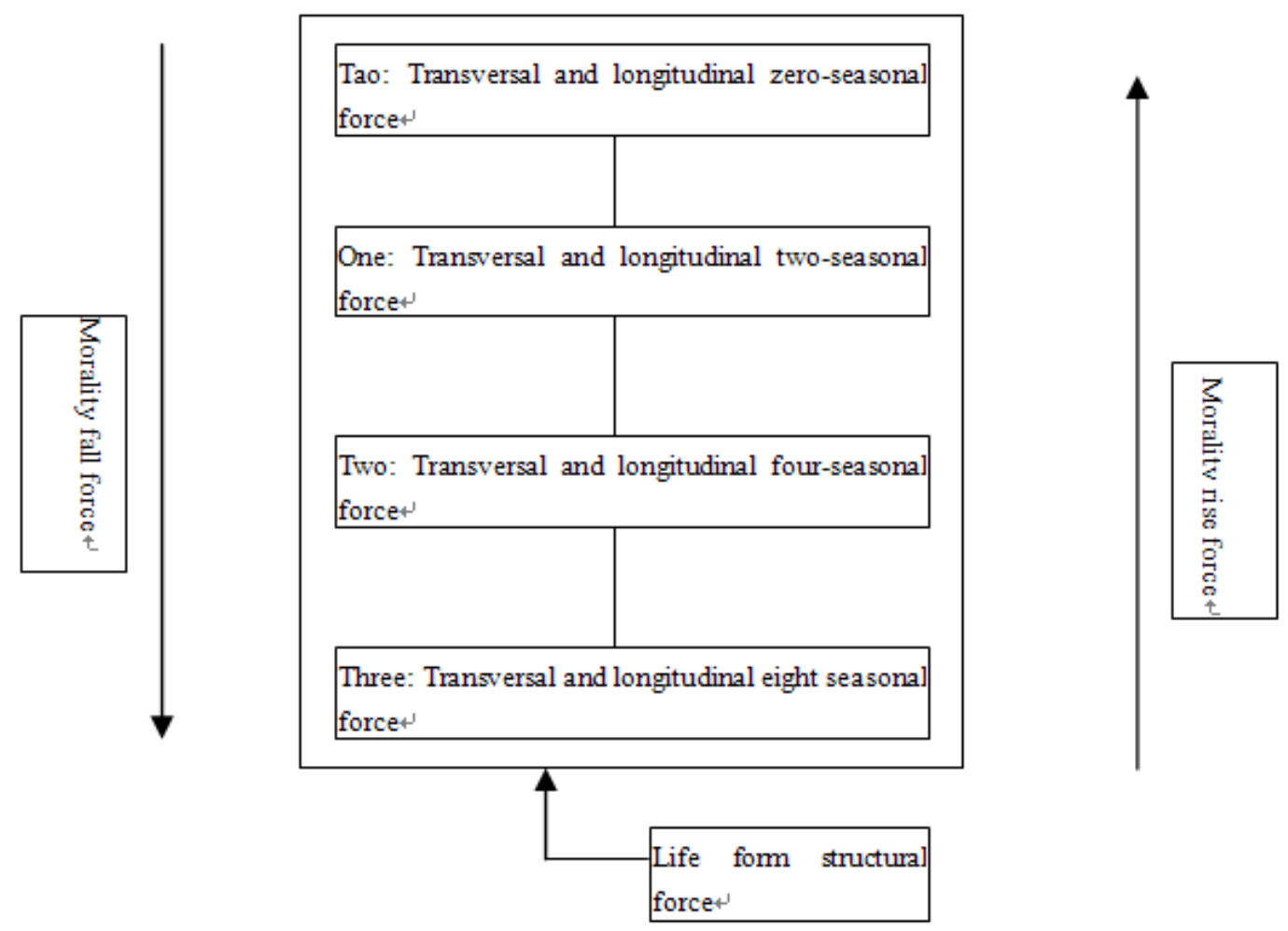

Fig. 3 Unity of all life form forces.

De is "one". "Two" is the highest morality energy and is the closest to Tao and Taoists call it mercy. "Three" has the lowest energy and includes justice, courtesy and wisdom.

\subsection{Moral Force and Morality Rise-Fall Force}

All things possess force as they have morality, which is called moral force. From Table 2, we can see the forces of things and the states of energy are identical to morality. That is to say, moral force is part of life forms' four-seasonal force.

Morality movement causes morality rise and fall, as Fig. 1 shows. Tao and De create two, and two create three, which is succession or morality fall, where energy falls and becomes visible. Three return to two, two return to one and one returns to zero, which is called reversion, where energy rises and become invisible. The rise or fall of life form internal energy is the moral rise-fall force.

The rise of internal moral energy is an energy gathering and causes cohesive force and rise in overall internal energy. The fall of moral energy is an energy scattering and causes dispersion force and splitting force. It is often seen in human society. Moral energy is also shown as attraction and repulsion. That is to say, having morality creates attraction and losing morality creates repulsion.

\subsection{Lack of Morality Is Abnormal}

The moral system of life consists of Tao, De, mercy, justice, courtesy, wisdom and trust. None of these energies can be absent and there must be abundant of them to ensure the normal movement of life forms. If one life form loses its moral energy or does not have enough moral energy, that is to say, it lacks some moral energy; thus, the four-seasonal movement of the life form will be out of balance or blocked and this life form must be abnormal [14].

\section{Thought Force}

Thought is an important system of life forms. Life forms have the capacity to think and this capacity is 
called thought force. The force or energy state of life forms determines their thought force or energy state. According to Form 2, brains at different energy states have different though elements and different thought states [11], namely different thought energies and thought forces. So thought force belongs to four-seasonal force.

Life movement includes thought movement. By controlling thought and breathing movement (Qigong and inner cultivation), we can increase our internal moral energy. Positive thought can increase internal energy while negative thought will decrease internal energy. That is to say, thought can control the rise and fall of human morality. So thought force is also morality rise-fall force.

Therefore, thought force is not only part of the four-seasonal force but also a morality rise-fall force.

\section{All Life Form Forces in the Universe Are Subject to General Law of the Universe}

See Fig. 3.

\section{Conclusions}

(1) All life form forces in the universe have the same structure and movement, namely four-seasonal structure, four-seasonal movement, four gradations and the rise and fall of the four gradations. This is the common feature of all life forms in the universe and also the general law of the universe.

(2) The unity of the universe includes the unity of all universal forces. Force combines, moves and changes things. Force includes binding force, structural force, moving force and changing force. Physical force (force of material) and life form force are the two kinds of universal force.

(3) Physical force (force of material) includes bonding force, strong interaction, quark confinement, weak interaction, electromagnetic interaction and gravitational interaction. Physical forces united into four-seasonal structure

(4) The force that exists inside life forms combines things together and pushes them to do four-seasonal movement or rise-fall movement is called life form force. It includes transversal four-seasonal force, longitudinal four-seasonal force, force within four gradation, integrating force and morality rise-fall force.

(5) Life form force is a compound, integral, long-range and automatically-closed four-seasonal force.

(6) The force that connects all parts of life forms and makes life forms integral bodies is the life form structural force. In the universe, this force is an invisible three-dimensional heavenly network, a rigid structure and movement. It makes all heavenly bodies in the universe have highly orderly structure and movement. Universal gravitation belongs to the life form structural force.

(7) The universe is a moral system and everything in it has morality. Moral movement can rise and fall, namely internal energy rise and fall. Morality has moral force which includes rise force and fall force. When humans lack morality, it is abnormal.

(8) Thought force is one feature of life form force and is represented as thought ability and energy as well as thought and morality rise and fall.

(9) All life form forces are subject to the general law of the universe.

\section{References}

[1] Yan, J. Z. 2015. "The Universal Grand Unified Theory Based on Life and Thought." Journal of Physical Science and Application 5 (2): 147-57.

[2] Lu, T., and Luo, L. F. 2005. Comments on Substances: From Electrons to Quarks. Beijing: Science Press, 160-83.

[3] Fang, L. T. 2013. Buddhist Philosophy. Beijing: Religon Culture Press, 126-69.

[4] Yan, J. Z. 1989. "Zero Space Theory." Qigong and Science 8 (6): 29.

[5] Lao, Zi. 2011. De Tao Ching. Beijing: The International Cultural Publishing Company, 89.

[6] Yan, J. Z. 2013. "Introduction to Unity of Physics and Biology, Inorganic Life Materials." Journal of Physical Science and Application 3 (1): 58-64. 
[7] Yan, J. Z. 2013. "State Space-Time and Four States of the Universe." Journal of Physical Science and Application 3 (2): 127-34.

[8] Yan, J. Z. 2014. "The First Push of the Universe and Origin of Life." Journal of Physical Science and Application 4 (3): 198-205.

[9] Yan, J. Z. 2013. "Automatic Organization, Thinking Motion and Dynamics of the Universe." Journal of Physical Science and Application 3 (3): 199-208.

[10] Zhang, Sh. J., and Yi, F. 2010. Revealing the Secret of
Horoscope. Beijing: China Business Press, 22-40.

[11] Xiong, Ch. J. 2011. Tao Medicine. Beijing: Tuanjin Press, $1-130$.

[12] Xiong, Ch. J. 2011. De Tao World. Beijing: China Yanshi Press, 60-9.

[13] Xiong, Ch. J. 2011. Civilization and Education of Loong Culture. Beijing: Tuanjie Press, 215-94.

[14] An anonymous author. 2013. The Observation Notes by High-Level Qigong. Guilin: Guangxi Normal University Press, 115. 